\title{
Étude de propriétés structurales et dynamiques par dynamique moléculaire ab initio : application aux verres et aux liquides vitreux
}

\author{
A. Pasquarello ${ }^{1,2}$ \\ ${ }^{1}$ Institut de Théorie des Phénomènes Physiques (ITP), École Polytechnique Fédérale \\ de Lausanne (EPFL), 1015 Lausanne, Suisse \\ ${ }^{2}$ Institut Romand de Recherche Numérique en Physique de Matériaux (IRRMA), \\ 1015 Lausanne, Suisse
}

\begin{abstract}
Résumé : La dynamique moléculaire $a b$ initio est une méthode de simulation dans laquelle l'évolution de la structure électronique est prise en compte explicitement. Après une brève description de la méthode, son application aux systèmes vitreux est illustrée à travers des études de $\mathrm{SiO}_{2}$ et de $\mathrm{GeSe}_{2}$. Dans le contexte des systèmes vitreux, les méthodes ab initio servent tout d'abord à la génération de modèles structuraux. Ensuite, ces méthodes peuvent être étendues à l'étude des propriétés vibrationnelles. Les méthodes $a b$ initio constituent aujourd'hui un outil indispensable permettant l'analyse d'une variété de propriétés expérimentales dans le contexte d'un seul cadre théorique cohérent.
\end{abstract}

\section{INTRODUCTION}

Les matériaux vitreux sont largement utilisés dans divers secteurs industriels et technologiques. De plus en plus, la nécessité de contrôler les propriétés de ces matériaux à l'échelle nanométrique exige une compréhension détaillée de leur structure atomique. A cause de l'absence d'ordre à longue portée, les méthodes expérimentales basées sur la diffraction de rayons $X$ ou de neutrons ne peuvent donner qu'une description partielle et incomplète de la structure atomique de ces matériaux. D'autres techniques expérimentales, telles que les spectroscopies vibrationnelles ou la résonance magnétique nucléaire, nous transmettent aussi des informations sur la structure atomique, mais leur interprétation est souvent difficile. Dans ce domaine, la simulation théorique est devenue ainsi un moyen essentiel pour raffiner nos connaissances. Le but ultime est de fournir des modèles structuraux avec des propriétés qui sont en bon accord avec toutes les données expérimentales disponibles. A terme, ceci implique que les méthodes théoriques devraient nous permettre d'établir un contact avec toutes les expériences qui pourraient contenir des informations structurales.

La structure atomique obtenue par une méthode de simulation dépend fortement de la qualité des interactions atomiques utilisées. Cette dépendance est particulièrement importante dans le cas d'un environnement désordonné, où les contraintes de périodicité sont absentes. Dans la dynamique moléculaire $a b$ initio [1], les interactions atomiques sont dérivées à partir d'une description quantique de la structure électronique, qui est à tout moment totalement cohérente avec les positions atomiques. La description des liaisons chimiques ne devrait donc pas être biaisée comme cela peut être le cas dans la dynamique moléculaire classique, où les interactions dérivent de potentiels qui n'ont été optimisés que pour un nombre limité de configurations. Cependant, la description améliorée de la liaison chimique dans les méthodes ab initio ne peut s'obtenir qu'au prix d'un coût en temps de calcul considérablement plus important. Ce coût augmente comme le nombre d'atomes au cube et représente aujourd'hui la principale raison qui empêche l'application des méthodes $a b$ initio à des systèmes de grande taille.

Ces dernières années, le développement de nouvaux algorithmes et la puissance croissante des ordinateurs ont permis aux méthodes $a b$ initio d'étendre leur rayon d'application au domaine 
des matériaux vitreux. Initialement, les applications les plus directes ont consisté à générer des modèles structuraux par dynamique moléculaire. Plus récemment, les méthodes ab initio ont aussi pu établir un contact avec les principales spectroscopies vibrationnelles, notamment, la diffusion inélastique de neutrons, l'absorption infrarouge, et la diffusion Raman.

Ce cours a pour but de donner une introduction à la méthode de dynamique moléculaire $a b$ initio et de démontrer son potentiel dans l'étude des matériaux vitreux à travers différentes applications récentes. La dynamique moléculaire $a b$ initio est une méthode de simulation qui réunit un calcul de la structure électronique dans le cadre de la théorie de la fonctionnelle de la densité et une dynamique moléculaire de type classique [1]. Le chapitre 2 donnera les bases théoriques de la méthode. D'abord, la théorie de la fonctionnelle de la densité sera brièvement rappelée. Ensuite, la discussion portera sur la Lagrangienne qui détermine l'évolution dans la dynamique moléculaire $a b$ initio. Le chapitre 3 sera consacré à la présentation d'applications qui concernent principalement des propriétés structurales. Les systèmes vitreux de $\mathrm{SiO}_{2}$ et de $\mathrm{GeSe}_{2}$ seront considérés comme exemples de systèmes avec liaisons chimiques contenant un degré de ionicité différent. Dans le chapitre 5 , nous verrons que les méthodes $a b$ initio permettent d'effectuer une comparaison directe avec les spectres vibrationnels obtenus par diffusion inélastique de neutrons, absorption infrarouge, ou diffusion Raman. Dans le chapitre 5, la discussion sera étendue à la description de propriétés électroniques. Les conclusions sont données au chapitre 6 .

\section{FORMULATION THEORIQUE}

La méthode présentée dans ce chapitre sera délimitée par deux importantes conditions. Premièrement, nous considérerons uniquement des cas où les positions nucléaires peuvent être traitées dans l'approximation classique. Deuxièmement, nous retiendrons l'approximation de Born-Oppenheimer, qui sépare le mouvement des atomes de l'état quantique des électrons. En pratique, pour chaque configuration nucléaire, les électrons sont à température $T=0 \mathrm{~K}$ et occupent leur état fondamental. Nous sommes intéressés par l'énergie de cet état et par les dérivées de son énergie par rapport aux déplacements nucléaires, qui déterminent les forces agissant sur les noyaux.

Plusieurs méthodes de calcul de la structure électronique, notamment celles développées en chimie quantique, pourraient donner une description fiable. Toutefois leur utilisation dans une dynamique moléculaire est conditionnée par leur coût numérique. La plupart de ces méthodes ont un coût qui varie comme le nombre d'atomes à la puissance quatre ou plus, qui est trop élevé pour être combiné avec une dynamique moléculaire, qui peut nécessiter le calcul de l'état électronique fondamental pour 10000 ou 100000 configurations nucléaires.

Un cadre acceptable est offert par la théorie de la fonctionnelle de densité. Dans ce cadre, le calcul de la structure électronique a un coût qui varie comme le nombre d'atomes à la troisième puissance. Plusieurs applications de cette théorie ont démontré qu'elle est capable de décrire avec une grande précision plusieurs propriétés de molécules, d'agrégats, et de matériaux cristallins.

\subsection{Théorie de la fonctionnelle de la densité}

La théorie de la fonctionnelle de la densité reconnaît en la densité életronique $n(\mathbf{r})$ une quantité qui détermine complètement l'état électronique fondamental. Cette constatation est importante puisqu'elle nous permet de définir un état quantique uniquement par la densité, fonction simple de la position $\mathbf{r}$, et d'éviter de nous référer à la fonction d'onde électronique $\Psi$, solution fondamentale de l'équation de Schrödinger. La fonction d'onde de Schrödinger dépend des degrés de liberté de tous les électrons, et des solutions pratiques n'existent que pour des cas à petit nombre d'électrons. Dans un important théorème, Hohenberg et Kohn [2] ont démontré que l'espérance de l'Hamiltonienne sur l'état fondamental peut être écrite en terme d'une fonctionnelle unique $F$ 
de la densité :

$$
\langle\Psi|H| \Psi\rangle=\langle\Psi|\hat{T}+\hat{W}+v(\mathbf{r})| \Psi\rangle=F[n]+\int d \mathbf{r} v(\mathbf{r}) n(\mathbf{r}),
$$

où $\hat{T}$ et $\hat{W}$ représentent respectivement l'opérateur d'énergie cinétique des électrons et l'opérateur d'interaction entre électrons, et où $v(\mathbf{r})$ est un potentiel externe. La fonctionnelle $F$ est unique dans le sens qu'elle ne dépend pas du potentiel externe qui agit sur les électrons. On entend par potentiel externe, un potentiel qui est externe au système électronique, comme par exemple le potentiel dérivant du champ Coulombien des noyaux. L'unicité de la fonctionnelle $F$ implique en particulier que la même fonctionnelle $F$ s'applique pour des électrons dans les atomes, les molécules, ou les solides.

Le deuxième résultat démontré par Hohenberg et Kohn [2] fournit un principe variationnel. Si nous définissons l'énergie d'un système de fermions soumis à un potentiel externe $v(\mathbf{r})$ par

$$
E_{v}[n]=F[n]+\int d \mathbf{r} v(\mathbf{r}) n(\mathbf{r}),
$$

l'état fondamental est atteint quand $E_{v}[n]$ est minimale par rapport aux variations de densité :

$$
E_{0}=\min _{n} E_{\nu}[n] \text {. }
$$

Dans cette équation, la densité électronique $n(\mathbf{r})$ peut varier dans une classe de densités dites $v$-représentables. Ces densités correspondent aux densités associées à des Hamiltoniennes $H^{\prime}=$ $\hat{T}+\hat{W}+v^{\prime}$, où $v^{\prime}$ est un potentiel externe local.

Les bases de la théorie de la fonctionnelle de la densité sont complétées par la contribution de Kohn et Sham [3]. Kohn et Sham ont montré qu'il est possible de décrire un système de fermions interagissant par un système auxiliaire décrivant un ensemble de particules non-interagissantes. A chaque électron est associé un état à une particule $\psi$. Les états $\psi$ sont orthonormalisés,

$$
\left\langle\psi_{i} \mid \psi_{j}\right\rangle=\delta_{i j}
$$

et la densité est définie en terme de ces états :

$$
n(\mathbf{r})=\sum_{i}^{\mathrm{occ}} f_{i}\left|\psi_{i}(\mathbf{r})\right|^{2}
$$

oì la sommation porte sur les états occupés et les $f_{i}$ représentent les occupations. Par simplicité, nous assumerons que les $f_{i}$ sont tous égaux (par exemple $f_{i}=2$, quand les effets de spin peuvent être négligés), et nous les négligerons par la suite. Nous utilisons ici des unités atomiques définies par $\hbar=m_{e}=e=1$. La fonctionnelle $F[n]$ est décomposée comme suit :

$$
F[n]=T[n]+\frac{1}{2} \int d \mathbf{r} d \mathbf{r}^{\prime} \frac{n(\mathbf{r}) n\left(\mathbf{r}^{\prime}\right)}{\left|\mathbf{r}-\mathbf{r}^{\prime}\right|}+E_{\mathrm{xc}}[n],
$$

où $T[n]$ correspond à l'énergie cinétique associée au système de particules non-interagissantes :

$$
T[n]=\sum_{i}\left\langle\psi_{i}\left|-\frac{1}{2} \nabla^{2}\right| \psi_{i}\right\rangle
$$

Le deuxième terme dans l'équation (6) représente l'énergie Coulombienne d'interaction entre électrons. Le troisième terme $E_{\mathrm{xc}}$ est appelé énergie d'échange et de corrélation, et représente toute contribution à l'énergie qui n'est pas prise en compte par les deux premiers termes. En fait, l'équation (6) n'est rien d'autre que la définition formelle de $E_{\mathrm{xc}}$. 
La minimisation de $E_{v}$ [équation (2)] peut être formulée en termes de variations des fonctions $\psi_{i}$ qui déterminent la densité $n$ par l'équation (5). En tenant compte de la condition d'orthonormalisation des $\psi_{i}$, nous obtenons ainsi les équations de Kohn et Sham [3] :

$$
\left[-\frac{1}{2} \nabla^{2}+\int d \mathbf{r}^{\prime} \frac{n\left(\mathbf{r}^{\prime}\right)}{\left|\mathbf{r}-\mathbf{r}^{\prime}\right|}+\frac{\delta E_{\mathbf{x c}}}{\delta n(\mathbf{r})}+v(\mathbf{r})\right] \psi_{i}=\epsilon_{i} \psi_{i}
$$

qui définissent l'Hamiltonienne de Kohn-Sham, $H_{\mathrm{Ks}} \psi_{i}=\epsilon_{i} \psi_{i}$. De cette manière, la théorie de la fonctionnelle de la densité transforme la recherche de l'état électronique fondamental d'un système de fermions interagissant en un problème d'équations couplées à une particule. La dérivation est purement formelle puisque la difficulté dans les équations (8) se cache dans le terme d'échange et de corrélation qui reste inconnu. Le succès de la théorie de la fonctionnelle de la densité est déterminé par le fait que des approximations relativement simples de $E_{\mathrm{xc}}$ ont donné des résultats très satisfaisants en comparaison avec l'expérience.

L'approximation la plus simple pour $E_{\mathrm{xc}}$ est dite approximation de la densité locale (LDA). Elle consiste à attribuer à chaque élément de volume unitaire centré autour de $\mathbf{r}$ et occupé par une densité $n(\mathbf{r})$ une énergie par électron égale à l'énergie par électron $e_{\mathrm{xc}}(\rho)$ que le volume aurait dans un gaz électronique homogène de densité constante $\rho=n(\mathbf{r})$ :

$$
E_{\mathrm{xc}}^{\mathrm{LDA}}[n]=\int d \mathbf{r} n(\mathbf{r}) e_{\mathrm{xc}}[n(\mathbf{r})] .
$$

L'énergie par électron $e_{\mathrm{xc}}(\rho)$ pour le gaz d'électrons a été calculée par une méthode Monte-Carlo [4] et a été utilisée longuement dans les calculs $a b$ initio basés sur la théorie de la fonctionnelle de la densité.

Aujourd'hui, Ies approximations les plus courantes pour le terme d'échange et de corrélation sont les approximations de gradient généralisées (GGA) qui ne dépendent pas que de la densité locale mais aussi de son gradient :

$$
E_{\mathrm{xc}}^{\mathrm{GGA}}[n]=\int d \mathbf{r} f_{\mathrm{xc}}[n(\mathbf{r}),|\nabla n(\mathbf{r})|] .
$$

Plusieurs fonctions $f_{\mathrm{xc}}$ sont utilisées aujourd'hui [5-7]. Les approximations de gradient généralisées donnent habituellement une meilleure description de l'énergie que l'approximation de densité locale [8].

\subsection{Calcul de structure électronique et d'énergie totale}

La théorie exposée dans la section précédente permet d'effectuer des calculs explicites de la structure électronique pour une configuration de noyaux donnée. Nous nous intéressons à l'énergie totale qui comprend un contribution électronique et une contribution nucléaire :

$$
\left.E^{\text {tot }}=E_{v}[n]+U\left(\left\{\mathbf{R}_{I}\right)\right\}\right),
$$

où $E_{v}$ est définie à partir de la théorie de la fonctionnelle de la densité et $U$ représente l'interaction Coulombienne répulsive entre les noyaux. L'interaction $U$ dépend uniquement des positions nucléaires et est une constante pour une configuration de noyaux donnée.

Concernant le calcul de la structure électronique, nous ne discutons ici que de la méthode qui est actuellement la plus utilisée en dynamique moléculaire. Elle est basée sur l'utilisation de pseudopotentiels et de bases d'ondes planes, en présence de conditions aux bords périodiques. Dans cette méthode, uniquement les électrons de valence sont traités explicitement. Il est donc assumé que les électrons de cœur ne participent pas à la formation des liaisons chimiques. Afin d'omettre complètement les électrons de cœur, les potentiels Coulombiens des noyaux sont remplacés par des potentiels effectifs, appelés pseudopotentiels, qui n'agissent que sur les électrons 
de valence, de telle manière à reproduire implicitement l'effet des électrons de cœur. Dans cette formulation, le système d'équations (8) ne se réfère qu'aux électrons de valence et le potentiel externe $v(\mathbf{r})$ devient :

$$
v(\mathbf{r})=\sum_{I} V^{I}\left(\mathbf{r}-\mathbf{R}_{I}\right)
$$

où la sommation porte sur les noyaux $I, \mathbf{R}_{I}$ représente la position du noyau $I$, et $V^{I}$ sont les pseudopotentiels. Les pseudopotentiels $V^{I}$ sont obtenus à partir de la structure électronique de l'atome isolé et contiennent généralement des termes non-locaux [9].

La dynamique moléculaire ab initio a utilisé longuement des pseudopotentiels qui conservent la propriété de normalisation des fonctions d'onde [9]. Toutefois, ce schéma exige des bases contenant un nombre très grand d'ondes planes pour traiter certains atomes du tableau périodique, notamment les atomes de la première rangée et les métaux de transition. Depuis une dizaine d'années, une nouvelle génération de pseudopotentiels a été implémentée avec succès dans le contexte de la dynamique moléculaire ab initio [10]. Désormais, tout atome du tableau périodique peut être traité avec un effort en temps de calcul comparable [11,12].

Les fonctions d'onde de valence $\psi_{i}$ sont développées sur une base d'ondes planes [13] :

$$
\psi_{i}(\mathbf{r})=\frac{1}{\sqrt{V}} \sum_{\mathbf{G}} c_{i, \mathbf{G}} e^{i \mathbf{G} \cdot \mathbf{r}},
$$

où $V$ est le volume de la cellule périodique. Dans ce développement, nous n'avons utilisé que le point $\Gamma$ de la zone de Brillouin. Cette approximation est souvent utilisée en pratique et se justifie par le fait que les systèmes étudiés en dynamique moléculaire sont désordonnés. Elle a comme conséquence que, grâce à la symétrie du point $\Gamma$, les fonctions d'onde peuvent être considérées réelles, permettant ainsi une économie importante de temps de calcul.

La solution des équations de Kohn-Sham (8) est trouvée pour des coefficients $c_{i, \mathrm{G}}$ qui minimisent la fonctionnelle $E_{v}[n]$. Il s'agit d'un problème de minimisation autocohérent. En fait, si nous admettons que la densité $n$ est connue, les équations (8) donnent un système linéaire pour les $c_{i, \mathrm{G}}$, qui peut être facilement résolu. Puisque en réalité $n$ dépend des fonctions d'onde et donc des $c_{i, \mathrm{G}}$, la solution est trouvée généralement par itération. Au départ, on utilise une densité approximative. La densité est ensuite progressivement améliorée en utilisant les fonctions d'onde de la dernière itération, jusqu'à ce que le processus converge. Les forces agissant sur les noyaux $I$,

$$
\mathbf{F}_{I}=-\frac{d E^{\mathrm{tot}}}{d \mathbf{R}_{I}}
$$

peuvent alors être calculées à partir des fonctions d'onde convergées. Dans la section suivante, nous verrons que la méthode Car-Parrinello peut être utilisée aussi bien pour la dynamique moléculaire que pour trouver la structure électronique dans une condition de noyaux immobiles.

\subsection{Dynamique moléculaire $a b$ initio}

En 1985, Car et Parrinello [1] ont été les premiers à présenter une dynamique moléculaire $a b$ initio, dans laquelle les forces utilisées dérivaient d'un traitement quantique des électrons, qui était à tout moment cohérent avec les positions nucléaires. Nous décrivons dans cette section les bases conceptuelles de cette méthode, qui sont demeurées inchangées jusqu'à aujourd'hui.

Il est utile d'initier la présentation par une variante de la dynamique moléculaire ab initio [14], qui a l'avantage d'être simple. Pour une configuration nucléaire donnée, on trouve d'abord l'état électronique fondamental et les forces nucléaires associées à cette configuration, suivant la démarche dans la section précédente. Ensuite, la configuration est évoluée dans le temps et l'opération est repétée pour la nouvelle configuration, et ainsi de suite. Cette méthode implique 
une nouvelle minimisation à chaque pas. Puisqu'un calcul numérique est toujours sujet à une erreur et que cette erreur entraine, dans le cas d'une minimisation, une surestimation systématique de l'énergie, la stabilité des quantités conservées par la dynamique moléculaire pourrait être compromise à long terme. Ces considérations nous portent à rechercher une méthode où toutes les variables sont véritablement dynamiques afin d'éviter toute minimisation. La méthode développée par Car et Parrinello satisfait précisément à ce critère.

L'idée principale de la méthode Car-Parrinello est d'associer une dynamique classique aux degrés de liberté qui caractérisent la structure électronique [1]. A chaque pas de l'évolution, la structure électronique est complètement déterminée par les fonctions d'onde, le cas échéant par les coefficients $c_{i, \mathrm{G}}$. Voulant associer une dynamique à ces coefficients, nous ne pouvons plus exiger la minimisation des fonctions d'onde à chaque pas de l'évolution. En d'autres termes, si les coefficients $c_{i, \mathrm{G}}^{0}$ correspondent à l'état fondamental électronique, il ne sera plus nécessaire que les variables dynamiques $c_{i, \mathrm{G}}$ soient égales aux $c_{i, \mathrm{G}}^{0}$, mais seulement qu'elles en restent suffisamment proches pendant tout le cours de l'évolution.

Formellement, ces conditions peuvent être résumées en un système Lagrangien qui comprend des degrés de liberté électroniques $\left\{\psi_{i}\right\}$ (en pratique il s'agit des $c_{i, \mathrm{G}}$ ) et des degrés de liberté nucléaires $\left\{\mathbf{R}_{I}\right\}$. La Lagrangienne qui détermine ce système classique est donnée par [1] :

$$
\mathcal{L}\left(\left\{\psi_{i}\right\},\left\{\psi_{i}^{*}\right\},\left\{\mathbf{R}_{I}\right\}\right)=\sum_{i} \mu\left\langle\dot{\psi}_{i} \mid \dot{\psi}_{i}\right\rangle+\frac{1}{2} M_{I} \dot{R}_{I}^{2}-E^{\text {tot }}\left(\left\{\psi_{I}\right\},\left\{\psi_{I}^{*}\right\},\left\{\mathbf{R}_{I}\right\}\right),
$$

où le premier terme correspond à l'énergie cinétique des degrés de liberté électroniques, le deuxième terme à l'énergie cinétique habituelle des noyaux, et le dernier terme à l'énergie totale définie dans la section précédente. Ce système Lagrangien est soumis aux contraintes d'orthonormalisation pour les fonctions d'onde données par l'équation (4), qui seront prises en compte en utilisant la méthode des paramètres de Lagrange. Notez, que le premier terme n'est pas physique et a été introduit pour donner une dynamique aux degrés de liberté électroniques. Une condition importante sera que ce premier terme, qui est connu sous le nom d'énergie cinétique fictive, reste toujours négligeable par rapport à l'énergie cinétique des noyaux. Cette condition peut être satisfaite en prenant la masse fictive $\mu$ suffisamment petite. Puisque $\mu$ détermine aussi l'échelle du temps des variations des $\left\{\psi_{i}\right\}$ et donc la longueur du pas d'intégration temporelle, il s'agira de trouver un bon compromis.

Dans l'équation (15), nous avons choisi de considérer comme variables indépendantes les fonctions d'onde $\psi_{i}$ et leurs complexes conjugués $\psi_{i}^{*}$, au lieu de leurs parties réelles et imaginaires. Ce choix nous amène aux équations d'Euler suivantes pour les fonctions d'onde :

$$
\mu \ddot{\psi}_{i}(\mathbf{r})=-\frac{\partial E_{v}}{\partial \psi^{*}(\mathbf{r})}+\sum_{i j} \Lambda_{i j} \psi_{j}(\mathbf{r})
$$

où $\Lambda_{i j}$ sont des paramètres de Lagrange dérivant de la condition d'orthonormalisation. Les équations d'Euler pour les $\psi_{i}^{*}$ donnent des équations qui sont les complexes conjugués des équations (16), et nous pouvons nous en passer pour le cas de fonctions d'onde réelles. Puisque la dérivée fonctionnelle

$$
\frac{\partial E_{v}}{\partial \psi^{*}(\mathbf{r})}=H_{\mathrm{Ks}} \psi_{i}
$$

où $H_{\mathrm{KS}}$ est l'Hamiltonienne de Kohn-Sham (8), les équations d'Euler pour les fonctions d'onde deviennent enfin :

$$
\mu \ddot{\psi}_{i}(\mathbf{r})=-H_{\mathrm{KS}} \psi_{i}+\sum_{i j} \Lambda_{i j} \psi_{j}(\mathbf{r})
$$

Les équations d'Euler pour les positions nucléaires $\mathbf{R}_{I}$ donnent :

$$
M_{I} \ddot{\mathbf{R}}_{I}=\mathbf{F}_{I}^{\mathrm{CP}}
$$


où

$$
\mathbf{F}_{I}^{\mathrm{CP}}=-\frac{\partial E^{\mathrm{tot}}}{\partial \mathbf{R}_{I}}
$$

sont les forces Car-Parrinello $\mathbf{F}_{I}^{\mathrm{CP}}$ agissant sur les positions nucléaires. Notez que les $\mathbf{F}_{I}^{\mathrm{CP}}$ deviennent identiques aux forces nucléaires $\mathbf{F}_{I}$ définies en (14) quand les fonctions d'onde correspondent à l'état électronique fondamental associé aux positions $\mathbf{R}_{I}$ instantanées. Cette condition est vérifiée seulement de manière approximative dans une évolution gouvernée par la Lagrangienne $\mathcal{L}$, puisque les fonctions d'onde évoluent dynamiquement en oscillant autour de l'état électronique fondamental. Toutefois, il a été démontré qu'en pratique les déviations des $\mathbf{F}_{I}^{\mathrm{CP}}$ par rapport aux $F_{I}$ sont minimes et de nature oscillatoire [15].

Les équations (16) et (19) définissent la dynamique moléculaire Car-Parrinello. Une condition importante est que les fonctions d'onde restent proches de l'état électronique fondamental à tout moment. En pratique, l'excitation des variables électroniques est empêchée par la présence d'une bande interdite entre les états électroniques occupés et vides [15]. Quand la bande interdite est mal définie ou absente comme dans les métaux, la méthode Car-Parinnello ne peut pas être appliquée directement et il devient nécessaire d'utiliser des solutions plus compliquées [16].

Il faut noter que la formulation de Car-Parrinello peut aussi être utilisée pour obtenir l'état électronique fondamental associé à des positions nucléaires fixées ou bien pour trouver une structure atomique relaxée. $\mathrm{Si}$, par exemple, pour des positions nucléaires fixées, on utilise l'évolution donnée par l'équation

$$
\mu \dot{\psi}_{i}(\mathbf{r})=-H_{\mathrm{KS}} \psi_{i}+\sum_{i j} \Lambda_{i j} \psi_{j}(\mathbf{r})
$$

le système des fonctions d'onde ne suivra plus une dynamique Newtonienne mais dissipera toute son énergie excédente jusqu'à ce que $\dot{\psi}_{i}=0$. Alors, les fonctions d'onde en (21) satisfont les équations de Kohn-Sham (8) à part une transformation unitaire qui diagonalise la matrice $\Lambda_{i j}$. De même, si l'équation (21) est utilisée en combinaison avec une équation de premier ordre pour les positions,

$$
M_{I} \dot{\mathbf{R}}_{I}=\mathbf{F}_{I}^{\mathrm{CP}}
$$

la structure atomique évoluera vers le minimum local d'énergie le plus proche.

Comme toute méthode de dynamique moléculaire, son implémentation pratique nécessite une discrétisation du temps et un algorithme d'intégration. Dans la dynamique moléculaire $a b$ initio, l'algorithme de Verlet est souvent utilisé car il garantit une bonne stabilité des quantités conservées. Dans le cas de la dynamique moléculaire, on obtient:

$$
\begin{aligned}
\psi_{i}(t+\Delta t) & \left.=2 \psi_{(} t\right)-\psi_{i}(t-\Delta t)-\frac{(\Delta t)^{2}}{\mu} H_{\mathrm{KS}} \psi_{i}(t)+\sum_{j} \Lambda_{i j} \psi_{j}(t) \\
\mathbf{R}_{I}(t+\Delta t) & =2 \mathbf{R}_{I}(t)-\mathbf{R}_{I}(t-\Delta t)+\frac{(\Delta t)^{2}}{M_{I}} \mathbf{F}_{I}^{\mathrm{CP}}(t)
\end{aligned}
$$

où $\Delta t$ est le pas de discrétisation du temps et les $\Lambda_{i j}$ sont obtenus en résolvant par itération les équations [17] :

$$
\left\langle\psi_{i}(t+\Delta t) \mid \psi_{j}(t+\Delta t)\right\rangle=\delta_{i j}
$$

qui imposent la condition d'orthonormalisation (4) au temps $t+\Delta t$. Des valeurs typiques pour $\Delta t$ se situent autour de $\sim 0.2 \mathrm{fs}$.

Finalement, nous mentionnons que le contrôle de la température s'effectue en couplant les équations à une variable additionnelle, qui contrôle directement l'énergie cinétique associée aux positions nucléaires [18]. En particulier, en utilisant ce couplage à température constante, la moyenne temporelle de toute quantité statique coïncide avec sa moyenne d'ensemble canonique. 
Une description plus approfondie de la méthode de la dynamique moléculaire ab initio est donnée dans la référence [19].

\section{PROPRIETES STRUCTURALES}

La dynamique moléculaire ab initio est une méthode très coûteuse du point de vue numérique. Ce coût numérique limite le nombre d'atomes indépendants qui peuvent être étudiés et la durée temporelle pendant laquelle leur mouvement peut être évolué. Avant de mettre en route une dynamique moléculaire, il est très important de comprendre quels sont les phénomènes physiques accessibles par leur étendue spatiale et leur durée temporelle. A l'état actuel, grâce aux algorithmes les plus récents et la puissance des derniers ordinateurs, nous pouvons envisager des simulations qui comprennent jusqu'à environ 200 atomes et qui évoluent pendant des périodes de quelques dizaines de picosecondes $[20,21]$.

La plupart des simulations $a b$ initio, dans le contexte des systèmes vitreux, concernent l'état liquide. Le système modelisé est porté à haute température et est laissé thermaliser. A l'équilibre thermique, la dynamique est utilisée pour obtenir des moyennes thermiques de propriétés physiques d'intérêt. En général, ce genre d'étude focalise sur les propriétés structurales qui peuvent être comparées aux résultats obtenus par diffraction de neutrons ou de rayons $X$. La comparajson entre propriétés mesurées et calculées nous donne une première indication de la qualité du modèle structural obtenu.

Des modèles de l'état vitreux peuvent être obtenus en baissant graduellement la température. Les temps de refroidissement dans la simulation sont très courts et diffèrent de plusieurs ordres de grandeur des temps expérimentaux correspondants. Donc, ces méthodes doivent être considérées comme des moyens artificiels pour obtenir des modèles structuraux, qui doivent par la suite être confirmés par la comparaison avec l'expérience. Toutefois, la possibilité même de générer des modèles structuraux, qui ne soient pas biaisés par des idées préçoncues, s'est révélée fort utile dans l'étude des verres, comme nous le verrons par la suite.

Dans ce chapitre, nous verrons des propriétés structurales qui peuvent être étudiées par la méthode de la dynamique moléculaire $a b$ initio dans le contexte des liquides vitreux. Nous

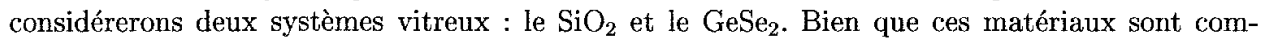
posés par des atomes qui appartiennent aux mêmes colonnes du tableau périodique, la différence d'électronégativité entre les atomes composants est beaucoup plus marquée pour le $\mathrm{SiO}_{2}$ que pour

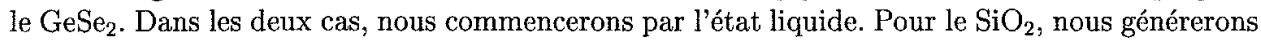
aussi un modèle de verre, qui sera utilisé par la suite dans l'étude des propriétés vibrationnelles.

\subsection{Dynamique moléculaire ab initio de $\mathrm{SiO}_{2}$}

Le liquide de $\mathrm{SiO}_{2}$ a été modelisé par un système périodique contenant 72 atomes à la densité expérimentale $[22,23]$. L'énergie d'échange et de corrélation a été décrite par l'approximation de densité locale. La température a été choisie en s'assurant que le système évolue effectivement comme un liquide. La relation d'Einstein nous dit que, dans un régime de diffusion, le déplacement carré moyen évolue linéairement en fonction du temps :

$$
\frac{1}{N_{\alpha}} \sum_{I=1}^{N_{\alpha}}\left|\mathbf{R}_{I}(t)-\mathbf{R}_{I}(t=0)\right|^{2}=6 D_{\alpha} t,
$$

où la sommation est effectuée sur les $N_{\alpha}$ atomes d'une espèce donnée et où $D_{\alpha}$ est le coefficient de diffusion respectif. Pour le liquide de $\mathrm{SiO}_{2}$, il a été nécessaire d'utiliser une température de 3500 $K$ afin d'observer suffisamment de diffusion. La figure 1 montre l'évolution du déplacement carré moyen pour les atomes de $\mathrm{Si}$ et de $\mathrm{O}$, qui nous permet de tirer une estimation pour la diffusion des ces deux espèces : $D_{\mathrm{Si}}=(5 \pm 1) \times 10^{-6} \mathrm{~cm}^{2} / \mathrm{s}$ pour le silicium et $D_{\mathrm{O}}=(9 \pm 2) \times 10^{-6} \mathrm{~cm}^{2} / \mathrm{s}$ 


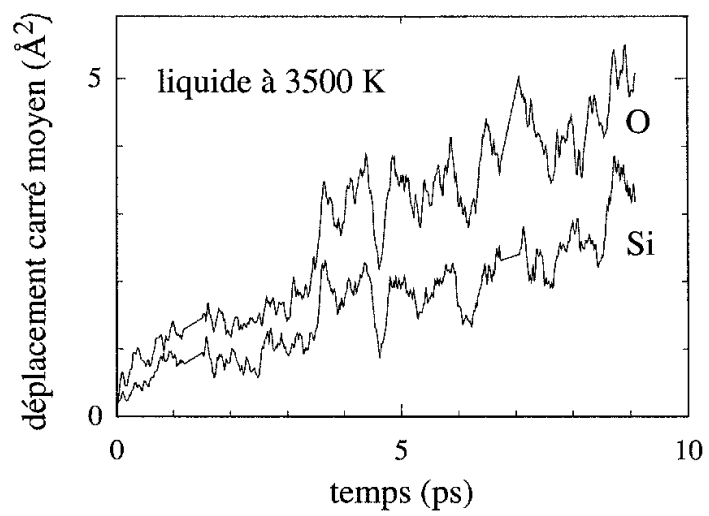

Figure 1 : Déplacement carré moyen pour la simulation de $\mathrm{SiO}_{2}$ liquide à une température de $3500 \mathrm{~K}$. Figure prise de la référence [23].

pour l'oxygène [23]. Après une évolution de l'ordre de $10 \mathrm{ps}$, la température à été baissée jusqu'à $300 \mathrm{~K}$ dans un temps d'environ $6 \mathrm{ps}$, obtenant ainsi un modèle structural pour le verre de $\mathrm{SiO}_{2}$.

Avant d'analyser en détail la structure obtenue, il est intéressant de comparer le facteur de structure dérivant de la simulation avec celui mesuré expérimentalement par diffraction de neutrons [24]. Le facteur de structure $S(\mathbf{Q})$ peut être calculé à partir des positions nucléaires $\mathbf{R}_{I}$ :

$$
S(\mathbf{Q})=\frac{1}{N\left\langle b^{2}\right\rangle} \sum_{I J} b_{I} b_{J}\left\langle e^{-i \mathbf{Q} \cdot\left(\mathbf{R}_{I}-\mathbf{R}_{J}\right)}\right\rangle
$$

où $N$ est le nombre d'atomes, où les parenthèses indiquent des moyennes thermiques, où les $b_{I}$ sont les longueurs de diffusion des neutrons, et où

$$
\left\langle b^{2}\right\rangle=\frac{1}{N} \sum_{I} b_{I}^{2}
$$

Le calcul de $\mathrm{S}(\mathbf{Q})$ est effectué pour tous les $\mathbf{Q}$ compatibles avec la périodicité de la cellule de simulation. Le résultat correspond donc au modèle infiniment répété. Pour des systèmes isotropiques $S(\mathbf{Q})$ ne dépend que du module de $\mathbf{Q}$. Pratiquement, $S(Q)$ est obtenu de $S(\mathbf{Q})$ par une moyenne sphérique [25]. La moyenne thermique est obtenue en considérant les configurations visitées dans le cours de la dynamique. Notez que les moyennes ainsi obtenues correspondent à un traitement classique des positions nucléaires.

La figure 2 montre les facteurs de structure pour deux systèmes désordonnés $\mathrm{de}_{\mathrm{SiO}_{2}}$ : le liquide à $3500 \mathrm{~K}$ et le modèle de verre à $300 \mathrm{~K}$. Expérimentalement, il n'a pas été possible d'obtenir le facteur de structure neutronique pour le liquide de $\mathrm{SiO}_{2}$ à cause des hautes températures. La comparaison à température ambiante entre le facteur de structure calculé et celui mesuré montre un très bon accord. Le facteur de structure a deux caractéristiques principales. La structure observée pour $Q>2 \AA^{-1}$ correspond à la formation d'unités tétraédriques de $\mathrm{Si}_{(}\left(\mathrm{O}_{4}\right)_{1 / 2}$. La simulation montre que, même à l'état liquide, le $\mathrm{SiO}_{2}$ est essentiellement composé d'unités tétraédriques. Le premier pic à $1.6 \AA^{-1}$ est caractéristique de systèmes désordonnés formant des réseaux relativement ouverts (pas trop denses) et indique la présence d'ordre à moyenne portée relatif à des distances dépassant la taille du tétraèdre [26]. Le bon accord avec l'expérience montre que, même à ces distances, l'ordre est bien reproduit par la simulation, malgré le petit nombre d'atomes dans le modèle. 


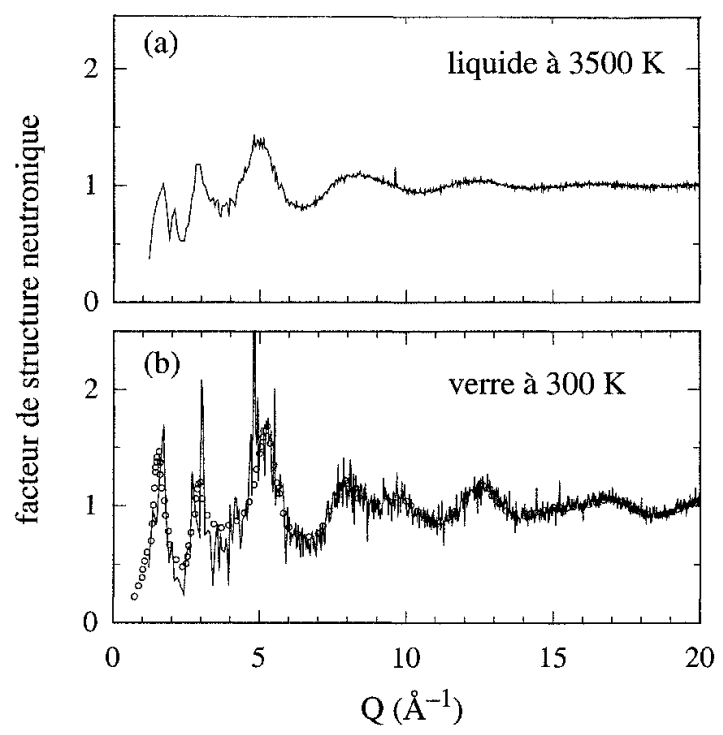

Figure 2 : Facteur de structure neutronique (a) pour le liquide de $\mathrm{SiO}_{2}$ à $3500 \mathrm{~K}$ et (b) pour le verre de $\mathrm{SiO}_{2}$ à $300 \mathrm{~K}$ (continu). Les cercles correspondent aux données expérimentales pour le verre de la référence [24]. Figure prise de la référence [22].

La figure 3 montre les distributions radiales associées aux corrélations $\mathrm{Si}-\mathrm{Si}, \mathrm{Si}-\mathrm{O}$, et $\mathrm{O}-\mathrm{O}$. Ces distributions ont été obtenues directement à partir des positions nucléaires, qui sont connues en détail dans une simulation de dynamique moléculaire. Les distributions radiales montrent clairement que l'ordre chimique est bien respecté dans le $\mathrm{SiO}_{2}$. En particulier, les premiers pics des corrélations $\mathrm{Si}-\mathrm{O}$ et $\mathrm{O}-\mathrm{O}$ correspondent bien à la formation d'unités tétraédriques. Expérimentalement, les distributions radiales peuvent être obtenues à partir des facteurs de structure partiels par transformation de Fourier. Dans le cas du verre de $\mathrm{SiO}_{2}$, les distributions radiales partielles n'ont pas encore pu être déterminées expérimentalement. Toutefois, les distributions dans la figure 3 montrent un bon accord avec les distributions radiales expérimentales obtenues pour le verre de $\mathrm{GeO}_{2}$ [27], qui a une structure proche à celle du verre de $\mathrm{SiO}_{2}$. Quand les distributions radiales théoriques et expérimentales sont comparées, il faut prêter attention au domaine de $Q$ qui a été utilisé dans la transformation de Fourier. En principe, les résultats expérimentaux sont seulement connus pour un domaine limité de $Q$, et, par conséquent, la distribution radiale ne pourra être qu'incomplète. Pour cette raison, il est préférable d'effectuer la comparaison entre théorie et expérience pour les facteurs de structure.

La disponibilité explicite des configurations atomiques dans la dynamique moléculaire, permet l'extraction d'autres propriétés structurales qui ne peuvent pas être obtenues directement par des méthodes de diffraction. Par exemple, la figure 4 donne les distributions angulaires $\mathrm{O}-\mathrm{Si}-\mathrm{O}$ et $\mathrm{Si}-\mathrm{O}-\mathrm{Si}$ entre premiers voisins pour le liquide de $\mathrm{SiO}_{2}$. La distribution O-Si-O est centrée à l'angle tétraédrique confirmant la présence d'unités $\mathrm{Si}\left(\mathrm{O}_{4}\right)_{1 / 2}$. La distribution angulaire $\mathrm{Si}-\mathrm{O}-\mathrm{Si}$, centrée autour de $130^{\circ}$, est plus large. Cette distribution caractérise le désordre en décrivant la disposition de tétraèdres voisins. Notez, que l'angle moyen trouvé pour le liquide $\left(134^{\circ}\right)$ est significativement inférieur aux angles typiques trouvés pour le verre à température ambiante $\left(\sim 150^{\circ}\right)$ [28-30]. 


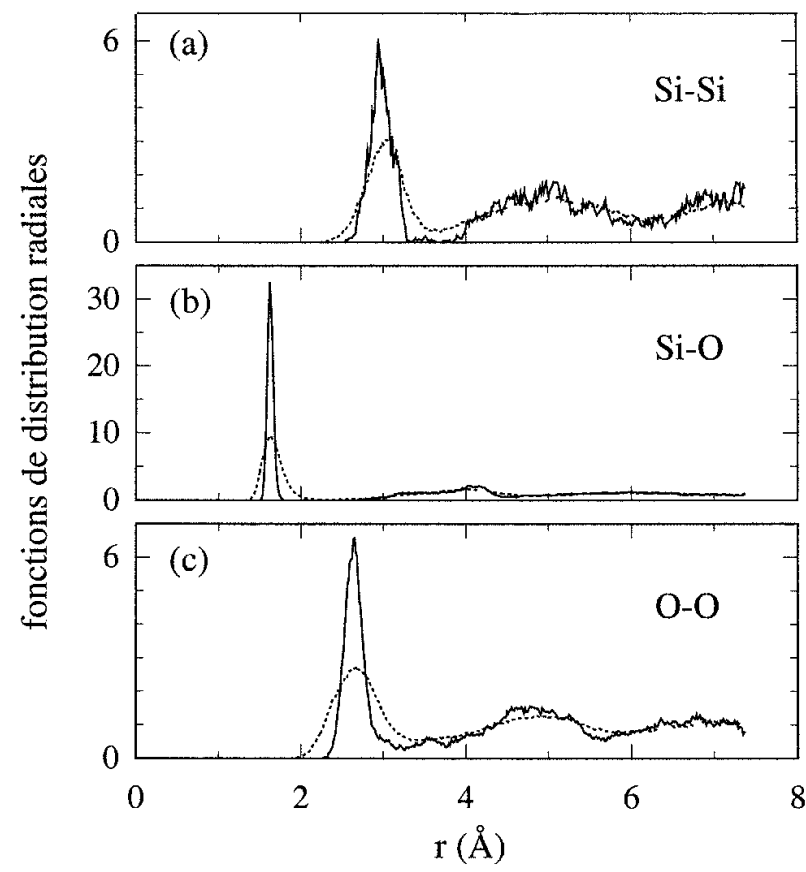

Figure 3 : Fonctions de distribution radiales pour le liquide de $\mathrm{SiO}_{2}$ à $3500 \mathrm{~K}$ (tirets) et pour le verre de $\mathrm{SiO}_{2}$ à $300 \mathrm{~K}$ (continu), relatives aux corrélations (a) $\mathrm{Si}-\mathrm{Si}$, (b) $\mathrm{Si}-\mathrm{O}$, et (c) O-O. Figure prise de la référence [23].

\subsection{Dynamique moléculaire ab initio de $\mathrm{GeSe}_{2}$}

Dans les systèmes désordonnés de $\mathrm{SiO}_{2}$, l'ordre chimique détermine un ordre à courte portée bien défini. Par contraste, dans le $\mathrm{GeSe}_{2}$, les électronégativités proches de Ge et Se admettent une plus grande variété de configurations structurales. Le liquide de $\mathrm{GeSe}_{2}$ a été étudié de façon approfondie aussi bien par diffraction de neutrons [31] que par dynamique moléculaire $a b$ initio [32-37]. En particulier, il a été possible d'obtenir une description structurale très détaillée. Les facteurs de structure partiels ont été mesurés par une technique expérimentale combinant la substitution isotopique et la diffraction neutronique [31].

Le liquide de $\mathrm{GeSe}_{2}$, modélisé par un système périodique de 120 atomes, a évolué à la température de $1040 \mathrm{~K}$ pour une durée dépassant les $20 \mathrm{ps}$. Afin de bien décrire les effets électroniques subtils liés à la présence d'électronégativités similaires pour Ge et $\mathrm{Se}$, il a été préféré d'utiliser une fonctionnelle GGA pour décrire l'énergie d'échange et de corrélation [6]. Dans le cours de la simulation, le déplacement carré moyen augmente de façon linéaire avec le temps, indiquant une diffusion typique de liquide. Les coefficients de diffusion obtenus pour les deux espèces atomiques sont pareils : $D=(2.2 \pm 0.2) \times 10^{-5} \mathrm{~cm}^{2} / \mathrm{s}[37]$.

Le facteur de structure dérivant de la simulation et celui mesuré par diffraction de neutrons sont comparés dans la figure 5. L'accord est excellent sur tout le domaine des $Q$. En particulier, la bonne description du premier pic de diffraction indique que même l'ordre sur des distances intermédiaires est à la portée des méthodes $a b$ initio. Une étude détaillée de la structure révèle que les unités tétraédriques de $\mathrm{Ge}\left(\mathrm{Se}_{4}\right)_{1 / 2}$ sont majoritaires, mais d'autres variétés structurales 


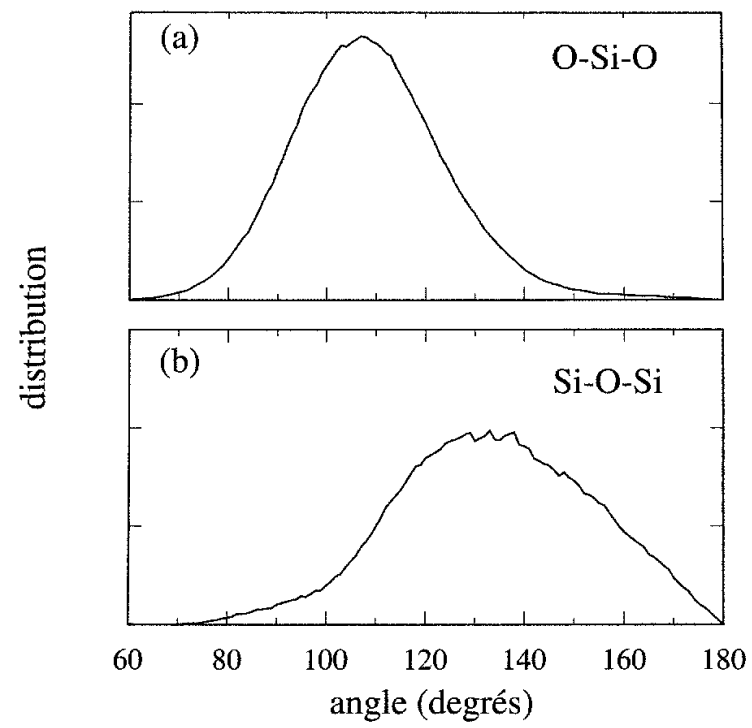

Figure 4 : Distributions des angles (a) $\mathrm{O}-\mathrm{Si}-\mathrm{O}$ et (b) $\mathrm{Si}-\mathrm{O}-\mathrm{Si}$ dans le liquide de $\mathrm{SiO}_{2}$ à $3500 \mathrm{~K}$. Figures prises de la référence [23].

sont aussi présentes. On observe, par exemple, des liaisons homopolaires $\mathrm{Ge}-\mathrm{Ge}$ et $\mathrm{Se}-\mathrm{Se}$, et un pourcentage non-négligeable d'atomes de Se et de Ge avec coordinence trois. La figure 6 montre une configuration instantanée du liquide pendant sa dynamique. Les différents motifs structuraux sont facilement identifiables. Cette description riche en variétés contraste avec la description obtenue par la dynamique moléculaire classique, où la structure est entièrement composée de tétraèdres [38].

La comparaison entre théorie et expérience peut être étendue aux facteurs de structure partiels. Dans la figure 7, les facteurs de structure partiels pour les corrélations Se-Se, Ge-Se et Ge-Ge, obtenus en utilisant les positions nucléaires dans la simulation, sont comparés à leurs correspondants expérimentaux [31]. L'accord est à nouveau très bon : seulement de petites différences sont observées pour les premiers pics de chaque facteur de structure [32].

Le liquide de $\mathrm{GeSe}_{2}$ a été utilisé souvent comme structure prototype afin d'étudier la formation d'ordre à moyenne portée. Grâce au bon accord entre théorie et expérience pour le premier pic dans la figure 5, la relation entre la manifestation de ce pic et la structure atomique peut être approfondie $[33,36]$. Il a été trouvé que la présence de ce pic dépend subtilement de la structure électronique. Une deuxième simulation effectuée en utilisant une fonctionnelle de la densité locale (LDA) a donné un facteur de structure sans premier pic de diffraction. Ce comportement a pu être rationalisé de la manière suivante. Le premier pic de diffraction a pu être lié à la présence d'une fraction importante de tétraèdres de $\mathrm{Ge}\left(\mathrm{Se}_{4}\right)_{1 / 2}$. Il a été démontré ensuite que la fonctionnelle GGA confère aux liaisons Ge-Se un caractère légèrement plus ionique que la fonctionnelle LDA, favorisant ainsi la formation de tétraèdres [33].

\section{PROPRIETES VIBRATIONNELLES}

Les méthodes $a b$ initio ont eu beaucoup de succès dans la description des bandes phononiques 


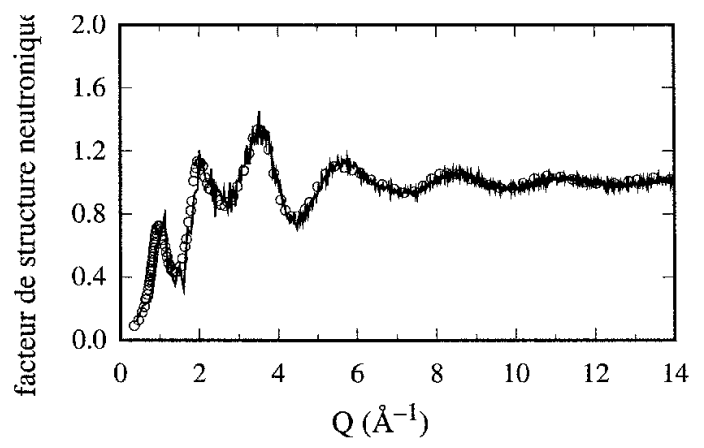

Figure 5 : Facteur de structure neutronique du liquide de $\mathrm{GeSe}_{2}$ à une température de $1040 \mathrm{~K}$. Les cercles correspondent aux données expérimentales de la référence [31]. Figure prise de la référence [32].

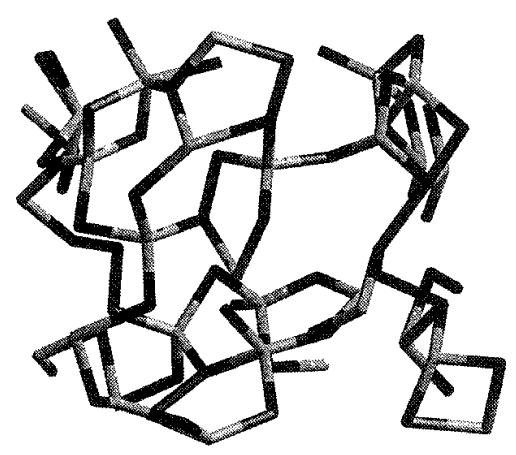

Figure 6 : Image représentant une configuration atomique instantanée du liquide de $\mathrm{GeSe}_{2}$ à une température de $1040 \mathrm{~K}$. Les bâtons noirs (gris) indiquent des demi-liaisons originaires d'atomes de Se (Ge). Deux atomes forment une liaison quand leur distance est inférieure à $3 \AA$. Figure prise de la référence [32].

des matériaux cristallins [39]. Il est de grand intérêt d'étendre ce genre de calculs aux propriétés vibrationnelles des verres. Les études de propriétés vibrationnelles de systèmes désordonnés par méthodes $a b$ initio sont restées rares, à cause de leur coût numérique. Nous verrons ici une étude de ce genre effectuée pour le verre de $\mathrm{SiO}_{2}[40-44]$.

En principe, la densité d'états vibrationnels peut être obtenue directement à partir d'une dynamique moléculaire en utilisant la fonction d'autocorrélation vitesse-vitesse. Toutefois, cette méthode se base sur un échantillonage ergodique de l'espace des phases, qui est souvent difficile à réaliser pour des systèmes fortement harmoniques. Pour le cas du verre de $\mathrm{SiO}_{2}$, il a été préféré de procéder par l'analyse des modes normaux d'une configuration d'équilibre. La matrice dynamique $D_{I \alpha, J \beta}$ de dimension $3 N \times 3 N$,

$$
D_{I \alpha, J \beta}=\frac{1}{\sqrt{M_{I} M_{J}}} \frac{d^{2} E^{\mathrm{tot}}}{d R_{I \alpha} d R_{J \beta}},
$$

où $N$ est le nombre d'atomes et $M_{I}$ la masse de l'atome $I$, caractérise les petites oscillations 


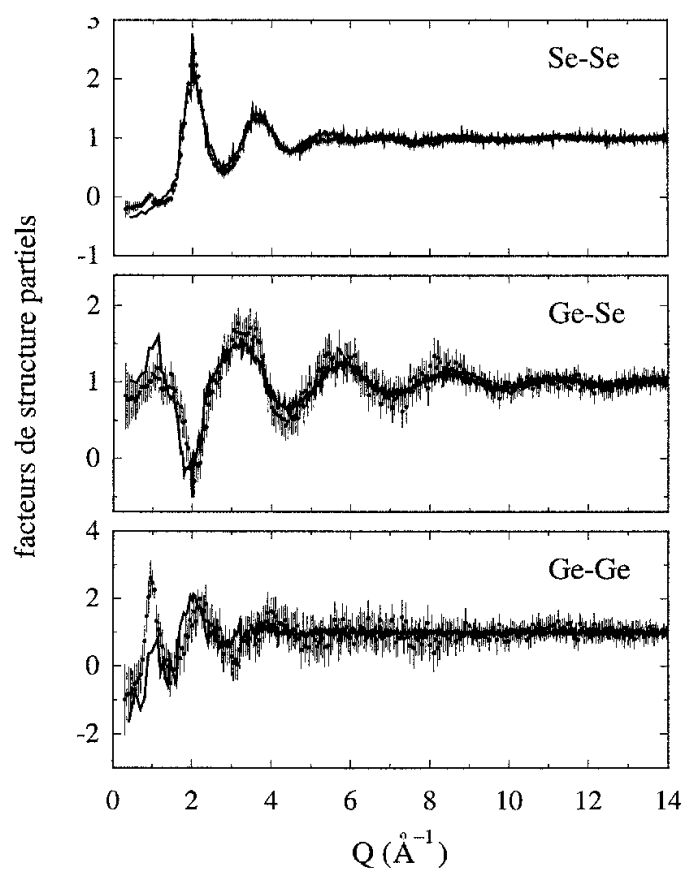

Figure 7 : Facteurs de structure partiels du liquide de $\mathrm{GeSe}_{2}$ à une température de $1040 \mathrm{~K}$ : Se-Se, $\mathrm{Ge}-\mathrm{Se}$, et Ge-Ge. Les barres verticales représentent les données expérimentales avec erreur de la référence [31]. Figure prise de la référence [32].

autour de l'équilibre. Elle peut être obtenue par l'application de la théorie des perturbations [39], ou, plus simplement, par une méthode de différences finies. C'est précisément cette dernière méthode qui a été utilisée afin de déterminer la matrice dynamique associée au modèle de verre de $\mathrm{SiO}_{2}$, généré préalablement par dynamique moléculaire ab initio. Dans cette méthode, la matrice dynamique est donnée par

$$
D_{I \alpha, J \beta}=-\frac{1}{\sqrt{M_{I} M_{J}}} \frac{\Delta F_{J \beta}^{I \alpha}}{\Delta R_{J \beta}}
$$

où $\Delta F_{J \beta}^{I \alpha}$ est la composante $\alpha$ de la force nucléaire agissant sur le noyau $I$, pour un déplacement $\Delta R_{J \beta}$ du noyau $J$ dans la direction $\beta(\alpha, \beta=x, y, z)$. Les calculs sont effectués dans un régime où les forces dépendent linéairement des déplacements. Les noyaux sont déplacés, l'un après l'autre, dans les trois directions Cartésiennes. Pour chaque déplacement, la structure électronique est trouvée et les forces (14) agissant sur tous les noyaux sont calculées, jusqu'à ce que toute la matrice soit déterminée. La diagonalisation de $D$ fournit alors les fréquences propres $\omega_{n}$ et les modes normaux correspondants $\boldsymbol{\xi}_{I}^{n}(n=1, . .3 N)$.

Les propriétés vibrationnelles sont généralement étudiées expérimentalement par trois techniques spectroscopiques différentes : la diffusion inélastique de neutrons, l'absorption infrarouge, et la diffusion Raman. La densité d'états vibrationnels $Z(E)$ est une quantité importante du 


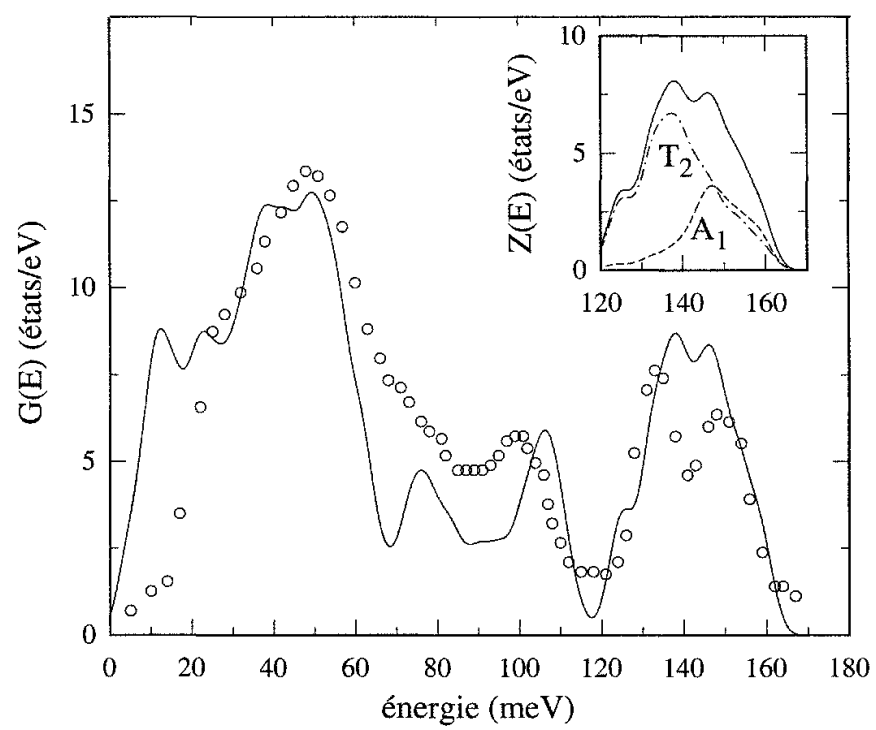

Figure 8 : Densité effective d'états vibrationnels $G(E)$ du verre de $\mathrm{SiO}_{2}$ calculée par des méthodes $a b$ initio (continu) et dérivée par diffusion inélastique de neutrons (cercles) [46]. En encadré : Densité d'états théorique $Z(E)$ décomposée selon les modes de symétrie du tétraèdre : $A_{1}$ et $T_{2}$. Figure reconstruite à partir des références $[40,43]$.

point de vue conceptuel et peut être obtenue facilement à partir des fréquences :

$$
Z(E)=\frac{1}{3 N} \sum_{n=1}^{3 N} \delta\left(E-\hbar \omega_{n}\right)
$$

Toutefois, elle ne peut pas être obtenue directement à partir de l'expérience. Nous focalisons ici sur des propriétés qui sont directement comparables aux résultats de mesures expérimentales.

La section efficace mesurée par diffusion inélastique de neutrons peut s'écrire :

$$
\frac{d^{2} \sigma}{d \Omega d E}=\frac{k_{1}}{k_{0}} S(\mathbf{Q}, E)
$$

où $k_{0}$ et $k_{1}$ sont les vecteurs d'onde du neutron entrant et sortant, où $\mathbf{Q}$ est le transfert de moment, où $E$ est l'énergie échangée entre le neutron et le matériau, et où $S(\mathbf{Q}, E)$ est la fonction de diffusion dynamique. A partir de la fonction $S(\mathbf{Q}, E)$ mesurée, il est possible de dériver une densité effective d'états vibrationnels $G(E)$, qui coïncide avec la densité $Z(E)$ seulement sous certaines conditions qui, en général, ne sont vérifiées qu'approximativement $[45,43]$.

Sous la condition qu'une seule excitation vibrationnelle est impliquée dans un évènement de diffusion, nous pouvons écrire $[45,43]$ :

$$
S(\mathbf{Q}, E)=\frac{1}{N\left\langle b^{2}\right\rangle} \sum_{I J} b_{I} b_{J} e^{i \mathbf{Q} \cdot\left(\mathbf{R}_{J}-\mathbf{R}_{I}\right)} \sum_{n} \hbar \frac{\left(\mathbf{Q} \cdot \boldsymbol{\xi}_{I}^{n}\right)^{*}\left(\mathbf{Q} \cdot \boldsymbol{\xi}_{J}^{n}\right)}{2 \omega_{n} \sqrt{M_{I} M_{J}}}\left[n\left(\hbar \omega_{n}\right)+1\right] \delta\left(E-\hbar \omega_{n}\right) .
$$

où $n\left(\hbar \omega_{n}\right)=\left[\exp \left(\hbar \omega / K_{B} T\right)-1\right]^{-1}$ est l'occupation Bosonique et où nous avons ornis par clarté les facteurs de Debye-Waller. La fonction $S(\mathbf{Q}, E)$ dans l'équation (33) peut être calculée explicitement pour un modèle donné en termes de ses fréquences propres et de ses modes normaux de 


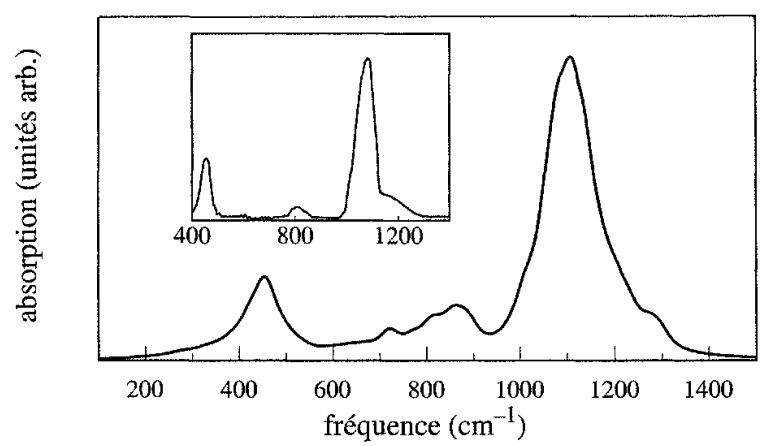

Figure 9 : Spectre d'absorption infrarouge du verre de $\mathrm{SiO}_{2}$, théorique et expérimental (en encadré) [49]. Figure prise de la référence [41].

vibrations $[40,43]$. Une fois obtenue la fonction $S(\mathbf{Q}, E)$, nous pouvons en tirer la densité effective $G(E)$ en empruntant le même procédé utilisé à partir de la fonction $S(\mathbf{Q}, E)$ mesurée.

Dans la figure 8, nous comparons la densité effective d'états vibrationnels $G(E)$ pour le verre de $\mathrm{SiO}_{2}$ dérivée à partir d'une diffusion inélastique de neutrons [46] avec sa correspondante calculée pour le modèle de verre généré par la dynamique moléculaire ab initio [22,23]. Malgré la petite taille du modèle, la théorie décrit correctement la position de tous les pics principaux, ainsi que leurs intensités relatives. En particulier, le doublet à hautes fréquences est reproduit par la théorie. L'origine de ce doublet a été longuement débattue dans la littérature. La qualité du spectre théorique et la disponibilité des modes normaux ont permis une analyse approfondie de l'origine de ce doublet $[40,43]$. Il a ainsi été possible de démontrer clairement que le doublet prenait son origine en des modes de vibrations associés à des représentations différentes de la symétrie du tétraèdre ( $A_{1}$ et $T_{2}$, voir encadré dans la figure 8). L'interprétation alternative basée sur un effet longitudinal-optique/transverse-optique (LO/TO) a pu être définitivement écartée. En fait, l'effet LO-TO apparaît seulement à petits $Q$ et s'annule dans la région des $Q$ mesurés dans l'expérience de diffusion de neutrons $[46,45]$.

Les spectres d'absorption infrarouge et de diffusion Raman ne peuvent être calculés directement à partir des vibrations mais nécessitent la connaissance additionnelle d'éléments de matrice spécifiques. Le spectre d'absorption infrarouge est lié à la partie imaginaire de la constante diélectrique transverse [41] :

$$
\operatorname{Im} \epsilon_{\perp}(\omega)=\frac{4 \pi^{2}}{3 \Omega} \sum_{n} \frac{\left|\mathbf{F}^{n}\right|^{2}}{2 \omega_{n}} \delta\left(\omega-\omega_{n}\right)
$$

Les forces d'oscillateurs $\mathbf{F}^{n}$ sont données par

$$
F_{\alpha}^{n}=\sum_{I} \sum_{\beta} Z_{\alpha \beta}^{I} \frac{\xi_{I \beta}^{n}}{\sqrt{m_{I}}}
$$

où les tenseurs $Z_{\alpha \beta}^{I}$ sont donnés par des matrices de dimension $3 \times 3$. Le tenseur $Z_{\alpha \beta}^{I}$ est connu sous le nom de charge dynamique de Born et détermine le couplage entre le déplacement nucléaire du noyau $I$ et le champ électrique. Pour une charge ponctuelle $q, Z$ est proportionnel au tenseur identité : $Z_{\alpha \beta}=q \delta_{\alpha \beta}$. Pour des électrons étendus, le calcul des tenseurs $Z_{\alpha \beta}^{I}$ nécessite la connaissance de la structure électronique. Ces tenseurs peuvent être obtenus en utilisant la théorie des perturbations [39] ou bien la théorie de la polarisation qui se base sur des concepts de phase 


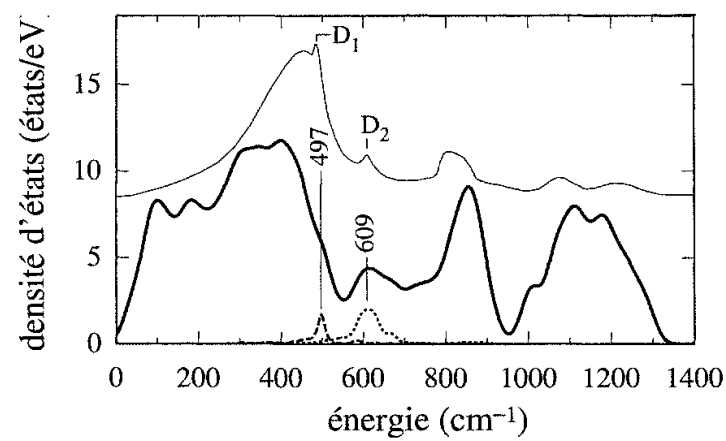

Figure 10 : Densité d'états vibrationnels du modèle de verre de $\mathrm{SiO}_{2}$ avec les projections sur les modes de vibration cohérents dans les anneaux à 6 (pointillé) et 8 (tirets) atomes. Le spectre Raman expérimental (trait fin) [53] est indiqué pour comparaison. Figure prise de la référence [42].

quantique $[47,48]$. Utilisant cette dernière théorie, il a été possible de déterminer les charges dynamiques pour les 72 atomes du modèle de verre de $\mathrm{SiO}_{2}$ [41]. Les charges dynamiques $Z_{\alpha \beta}$ pour les atomes de silicium sont bien décrites par des charges ponctuelles distribuées autour de 3.2 , en unités de charges électroniques $|e|$. Les charges dynamiques des atomes d'oxygène ont aussi une partie dominante qui agit comme charge ponctuelle (environ -1.6), mais elles comportent aussi d'autres contributions non-négligeables. Le fait que les charges dynamiques de $\mathrm{Si}$ et $\mathrm{O}$ diffèrent significativement de +4 et -2 , respectivement, indique que les liaisons $\mathrm{Si}-\mathrm{O}$ dans le verre de $\mathrm{SiO}_{2}$ ne peuvent être considérés de nature purement ionique.

Nous avons maintenant tous les ingrédients pour calculer le spectre d'absorption infrarouge du verre de $\mathrm{SiO}_{2}$. La figure 9 montre la comparaison entre le spectre calculé [41] et le spectre mesuré [49]. L'accord est très satisfaisant : les intensités relatives calculées des trois pics correspondent bien à leurs valeurs expérimentales. Il faut noter que des approches basées sur des méthodes empiriques n'arrivent pas à décrire correctement la distribution des intensités dans le spectre [50], la raison étant que les contributions non-ponctuelles aux charges dynamiques des atomes d'oxygène ne sont pas correctement prises en compte. Si l'on néglige ces contributions, les rapports entre les intensités des pics changent [41], notamment, l'intensité du pic à plus basse fréquence augmente tandis que celle du pic à plus haute fréquence diminue.

Dans un processus Stokes de diffusion Raman, un photon entrant de fréquence $\omega_{\mathrm{L}}$ et de polarisation $\hat{\mathrm{e}}_{\mathrm{L}}$ donne lieu à un photon sortant avec fréquence $\omega_{\mathrm{S}}$ et polarisation $\hat{\mathrm{e}}_{\mathrm{S}}$ et à une excitation vibrationnelle de fréquence $\omega_{k}$. La section efficace peut s'écrire [51] :

$$
\frac{d^{2} \sigma}{d \Omega d E}=\frac{\omega_{\mathrm{S}}^{4} V}{(4 \pi)^{2} c^{4}} \sum_{k}\left|\hat{\mathbf{e}}_{\mathrm{S}} \cdot \alpha^{k} \cdot \hat{\mathbf{e}}_{\mathrm{L}}\right|^{2} \frac{\hbar}{2 \omega_{k}}\left[n\left(\hbar \omega_{k}\right)+1\right] \delta\left(E-\hbar \omega_{k}\right)
$$

où $V$ est le volume, $c$ la vitesse de la lumière, $E$ l'énergie échangée, et où $\alpha^{k}$ est la susceptibilité Raman associée au mode normal $k$ :

$$
\alpha_{\mu \nu}^{k}=\sqrt{V} \sum_{I \gamma} \frac{d \chi_{\mu \nu}}{d R_{I \gamma}} \frac{\xi_{I \gamma}^{k}}{\sqrt{M_{I}}}
$$

où $\chi_{\mu \nu}$ est le tenseur de polarisabilité électrique. Afin de calculer des intensités Raman, il est donc nécessaire de connaître les tenseurs $d \chi_{\mu \nu} / d R_{I \gamma}$. Il existe des applications à des systèmes cristallins où ces tenseurs ont été calculés dans le cadre de la théorie de la fonctionnelle de la 


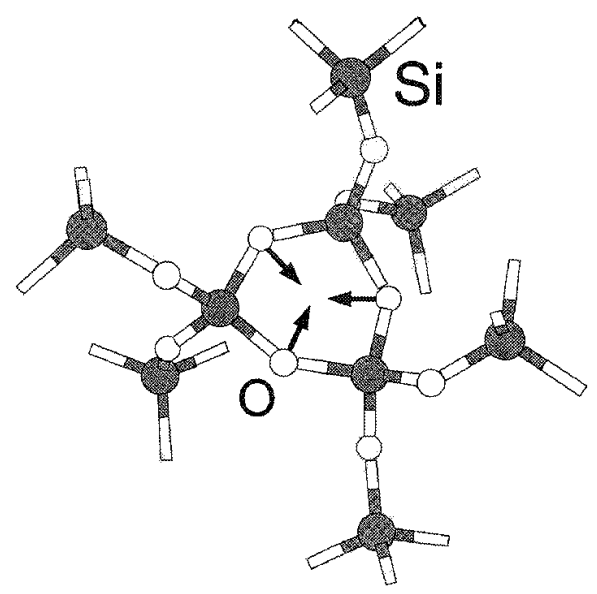

Figure 11 : Anneau de six atomes immergé dans la structure désordonnée de $\mathrm{SiO}_{2}$. Les flèches indiquent la direction du mouvement vibrationnel des atomes d'oxygène. Figure prise de la référence [42].

densité. Par exemple, les intensités Raman calculées pour le $\alpha$-quartz se comparent bien avec les intensités Raman mesurées [52]. Toutefois, des spectres Raman $a b$ initio relatifs à des systèmes désordonnés ne sont pas encore parus dans la littérature, à cause de leur coût numérique élevé.

Pour le verre de $\mathrm{SiO}_{2}$, il a toutefois été possible d'interpréter certaines propriétés du spectre Raman grâce à la connaissance des modes vibrationnels [42]. Le spectre expérimental Raman montre deux pics étroits, $D_{1}$ et $D_{2}$, aux fréquences de 495 et $606 \mathrm{~cm}^{-1}$ (figure 10). L'origine de ces pics a été longuement discutée dans la littérature. Une des explications qui ont été proposées consiste à attribuer leur origine à des anneaux de petite taille [53]. Il a été imaginé que les anneaux contenant 6 et 8 atomes soient planaires et que leurs atomes d'oxygène vibrent en phase le long des bisectrices des angles $\mathrm{Si}-\mathrm{O}-\mathrm{Si}$ (figure 11). Cette conjecture a été examinée de manière critique en utilisant le modèle du verre de $\mathrm{SiO}_{2}$ décrit précédemment $[22,23]$. La figure 10 montre les résultats des projections de modes normaux vibrationnels le long des mouvements atomiques proposés. Les projections ont donné des pics localisés indiquant des fréquences précises : 497 et $609 \mathrm{~cm}^{-1}$ pour les vibrations dans les anneaux de 8 et 6 atomes, respectivement, en excellent accord avec les positions expérimentales des pics $D_{1}$ et $D_{2}$. Ce bon accord permet d'affirmer que l'origine de ces pics Raman est maintenant comprise. Prochainement, le calcul ab initio des intensités Raman associées à ces deux pics nous permettra d'extraire les concentrations d'anneaux de petite taille dans le verre de $\mathrm{SiO}_{2}[54]$.

\section{PROPRIETES ELECTRONIQUES}

La structure électronique est constamment calculée dans le cours de la simulation de dynamique moléculaire $a b$ initio. En particulier, la densité d'états électroniques peut être obtenue sans aucun effort important supplémentaire. Dans le contexte des simulations de matériaux vitreux, la densité d'états électroniques a été utilisée pour interpréter $[22,23]$ les différences entre les spectres de photoémission du verre de $\mathrm{SiO}_{2}$ et de l' $\alpha$-quartz [55]. Les spectres théoriques dans la figure 12 ont été obtenus par dynamique moléculaire $a b$ initio à une température de $300 \mathrm{~K}$.

Nous avons vu dans le chapitre précédent que le calcul des intensités infrarouges et Raman ne pouvaient se faire sans avoir une description de la structure électronique. La connaissance 


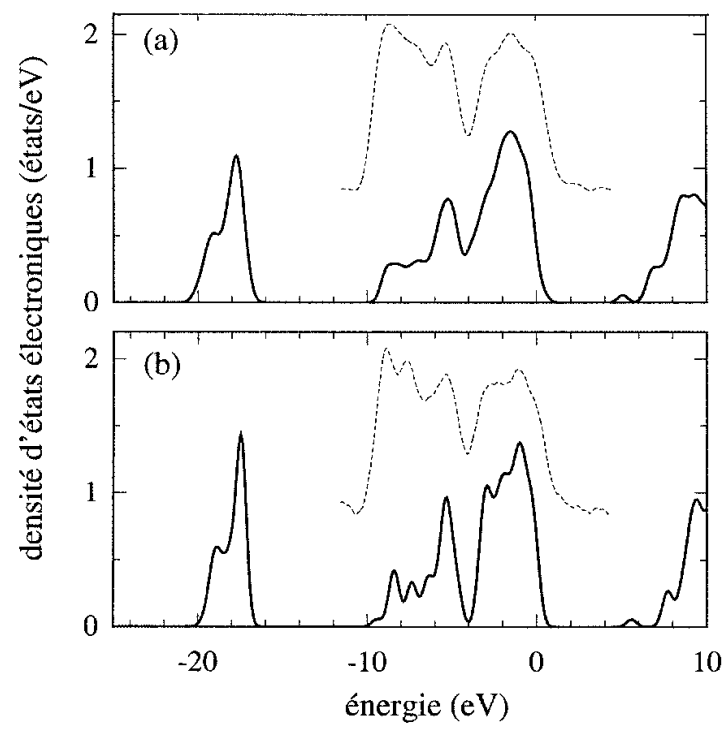

Figure 12 : Densité d'états électroniques (continu) (a) pour le verre de $\mathrm{SiO}_{2}$ à $300 \mathrm{~K}$ et (b) pour $\alpha$-quartz. Les données expérimentales de photoémission (tirets) proviennent de la référence [55]. Figure prise de la référence [22].

de la structure électronique est également à la base d'une méthode $a b$ initio pour le calcul des décalages chimiques [56], observés par résonance magnétique nucléaire (NMR). Dans une application récente de cette théorie [30], la distribution des angles $\mathrm{Si}-\mathrm{O}-\mathrm{Si}$ dans le verre de $\mathrm{SiO}_{2}$ a pu être extraite du spectre expérimental de NMR.

Il est facile d'anticiper que les propriétés électroniques des systèmes vitreux acquerront davantage d'intérêt dans le futur proche. Dans le contexte de la dynamique moléculaire ab initio cette tendance est illustrée par une récente application au ion hydraté $\mathrm{Cu}$ (II) [21], dans laquelle la densité conjointe d'états occupés et vides ainsi que le spectre XANES ont été obtenus en moyennant les résultats de multiples configurations atomiques.

\section{CONCLUSION}

Les méthodes de simulation $a b$ initio, comme la dynamique moléculaire $a b$ initio, permettent désormais une étude approfondie des systèmes vitreux, offrant un cadre théorique dans lequel plusieurs données expérimentales peuvent être interprétées de façon cohérente. La possibilité d'avoir accès à une description explicite de la structure électronique est à la base de la versatilité de ces méthodes. Actuellement, le coût numérique de ces approches est le facteur principal qui empêche un usage généralisé. Toutefois, cette situation évolue continuellement grâce aux développements de nouveaux algorithmes et à la disponibilité d'ordinateurs de plus en plus puissants.

\section{Remerciements.}

L'auteur remercie tous ses collaborateurs dans ce domaine de recherche : P. Umari, J. Sarnthein, C. Massobrio, et R. Car. Il remercie également I. Alméras Pasquarello et H.E. Fischer 
pour une lecture approfondie du manuscrit. Finalement, il remercie pour leur soutien le Fonds national suisse pour la recherche scientifique (Subside No. 620-57850.99) et le Centre suisse de calcul scientifique (CSCS).

\section{Références}

1. R. Car, M. Parrinello, Phys. Rev. Lett. 55, 2471 (1985).

2. P. Hohenberg, W. Kohn, Phys. Rev. B136 , 864 (1964).

3. W. Kohn, L.J. Sham, Phys. Rev. A140, 1133 (1965).

4. D.M. Ceperley, B.J. Alder, Phys. Rev. Lett. 45, 566 (1980).

5. A.D. Becke, Phys. Rev. A 38, 3098 (1988); C.Lee, W. Yang, R.G. Parr, Phys. Rev. B 37, 785 (1988).

6. J.P Perdew, Y. Wang, Phys. Rev. B 45, 13244 (1992)

7. J.P. Perdew, K. Burke, M. Ernzerhof, Phys. Rev. Lett. 77, 3865 (1996).

8. A. Dal Corso, A. Pasquarello, A. Baldereschi, R. Car, Phys. Rev. B 53, 1180 (1996).

9. D.R. Hamann, M. Schlüter, C. Chiang, Phys. Rev. Lett. 43, 1494 (1979).

10. D. Vanderbilt, Phys. Rev. B 41, 7892 (1990).

11. A. Pasquarello, K. Laasonen, R. Car, C. Lee, D. Vanderbilt, Phys. Rev. Lett. 69, 1982 (1992).

12. K. Laasonen, A. Pasquarello, R. Car, C. Lee, D. Vanderbilt, Phys. Rev. B 47, 10142 (1993).

13. J. Ihm, A. Zunger, M.L. Cohen, J. Phys. C : Solid State Phys. 12, (1979).

14. M.C. Payne, M.P. Teter, D.C. Allan, T.A. Arias, J.D. Joannopoulos, Rev. Mod. Phys. 64, 1045 (1992).

15. G. Pastore, E. Smargiassi, F. Buda, Phys. Rev. A 44, 6334 (1991).

16. P.E. Blöchl, M. Parrinello, Phys. Rev. B 45, 9413 (1992).

17. J. P. Ryckaert, G. Ciccotti, H. J. C. Berendsen, J. Comput. Phys. 23, 327 (1977) ; G. Ciccotti, J. P. Ryckaert, Computer Physics Reports 4, 345 (1986).

18. S. Nosé, Mol. Phys. 52, 255 (1984); W.G. Hoover, Phys. Rev. A 31, 1695 (1985).

19. G. Galli, A. Pasquarello, in Computer Simulation in Chemical Physics, edited by M. P. Allen and D. J. Tildesley (Kluwer, Dordrecht, 1993), p. 261.

20. A. Pasquarello, M.S. Hybertsen, R. Car, Nature 396, 58 (1998).

21. A. Pasquarello, I. Petri, P.S. Salmon, O. Parisel, R. Car, E. Tóth, D.H. Powell, H.E. Fischer, L. Helm, A.E. Merbach, Science 291, 856 (2001).

22. J. Sarnthein, A. Pasquarello, R. Car, Phys. Rev. Lett. 74, 4682 (1995).

23. J. Sarnthein, A. Pasquarello, R. Car, Phys. Rev. B 52, 12690 (1995).

24. S. Susman, K.J. Volin, D.L. Price, M. Grimsditch, J.P. Rino, R.K. Kalia, P. Vashishta, Phys. Rev. B 43, 1194 (1991).

25. D.L. Price, A. Pasquarello, Phys. Rev. B 59, 5 (1999).

26. S.R. Elliott, Nature 354, 445 (1991).

27. D.L. Price, M.-L. Saboungi, A.C. Barnes, Phys. Rev. Lett. 81, 3207 (1998).

28. R.L. Mozzi, B.E. Warren, J. Appl. Crystallogr. 2, 164 (1969). 
29. E. Dupree, R.F. Pettifer, Nature 308, 523 (1984).

30. F. Mauri, A. Pasquarello, B.G. Pfrommer, Y.-G. Yoon, S.G. Louie, Phys. Rev. B 62, R4786 (2000).

31. I.T. Penfold, P.S. Salmon, Phys. Rev. Lett. 67, 97 (1991).

32. C. Massobrio, A. Pasquarello, R. Car, Phys. Rev. Lett. 80, 2342 (1998).

33. C. Massobrio, A. Pasquarello, R. Car, J. Am. Chem. Soc. 121, 2943 (1999).

34. C. Massobrio, A. Pasquarello, R. Car, Comp. Mat. Science 17, 115 (2000).

35. C. Massobrio, F. H. M. van Roon, A. Pasquarello, S. W. De Leeuw, J. Phys. : Condens. Matter 12, L697 (2000).

36. C. Massobrio, A. Pasquarello, J. Chem. Phys. 114, 7976 (2001).

37. C. Massobrio, A. Pasquarello, R. Car, Phys. Rev. B 64, 144205 (2001).

38. P. Vashishta, R.K. Kalia, I. Ebbsjö, Phys. Rev. B 39, 6034 (1989).

39. S. Baroni, S. de Gironcoli, A. Dal Corso, P. Giannozzi, Rev. Mod. Phys. 73, 515 (2001).

40. J. Sarnthein, A. Pasquarello, R. Car, Science 275, 1925 (1997).

41. A. Pasquarello, R. Car, Phys. Rev. Lett. 79, 1766 (1997).

42. A. Pasquarello, R. Car, Phys. Rev. Lett. 80, 5145 (1998).

43. A. Pasquarello, J. Sarnthein, R. Car, Phys. Rev. B 5714133 (1998).

44. A. Pasquarello, Phys. Rev. B 61, 3951 (2000).

45. D.L. Price, J.M. Carpenter, J. Non-Cryst. Solids 92, 153 (1987).

46. J.M. Carpenter, D.L. Price, Phys. Rev. Lett. 54441 (1985).

47. R.D. King-Smith, D. Vanderbilt, Phys. Rev. B 47, 1651 (1993).

48. R. Resta, J. Phys. : Condens. Matter 12, R107 (2000).

49. C.T. Kirk, Phys. Rev. B 38, 1255 (1988).

50. M. Wilson, P.A. Madden, M. Hemmati, C.A. Angell, Phys. Rev. Lett. 77, 4023 (1996).

51. Light Scattering in Solids II, edited by M. Cardona and G.Güntherodt (Springer-Verlag, Berlin, 1982).

52. P. Umari, A. Pasquarello, Phys. Rev. B 63, 094305 (2001).

53. F.L. Galeener, Solid State Commun. 44, 1037 (1982); J. Non-Cryst. Solids 49, 53 (1982).

54. P. Umari, A. Pasquarello, Physica B : Condensed Matter 316-317, 572 (2002) ; P. Umari, A. Pasquarello, to appear.

55. B. Fischer, R.A. Pollak, T.H. DiStefano, W.D. Grobman, Phys. Rev. B 15, 3193 (1977); R.B. Laughlin, J.D. Joannopoulos, D.J. Chadi, Phys. Rev. B 20, 5228 (1979).

56. F. Mauri, B.G. Pfrommer, S.G. Louie, Phys. Rev. Lett. 77, 5300 (1996). 